\title{
The Chern Classes of Sobolev Connections
}

\author{
Karen K. Uhlenbeck
}

Department of Mathematics, University of Chicago, Chicago, Illinois 60637, USA

\begin{abstract}
Assume $F$ is the curvature (field) of a connection (potential) on $\mathbf{R}^{4}$ with finite $L^{2}$ norm $\left(\int_{\mathbf{R}^{4}}|F|^{2} d x<\infty\right)$. We show the chern number $c_{2}=1 / 8 \pi^{2} \int_{\mathbf{R}^{4}} F \wedge F$ (topological quantum number) is an integer. This generalizes previous results which showed that the integrality holds for $F$ satisfying the Yang-Mills equations. We actually prove the natural general result in all even dimensions larger than 2.
\end{abstract}

\section{Introduction}

All solutions of the Yang-Mills equations on $\mathbf{R}^{4}$ with finite action actually arise from connections defined on $\mathbf{R}^{4} \cup(\infty)=S^{4}$ [1,2]. This implies that the chern numbers of these connections are the chern numbers of a bundle over $S^{4}$, and hence are integers. It seems to be a question of general interest whether this result holds for arbitrary connections on $\mathbf{R}^{4}$ with finite energy [3]. Schlafley showed this is indeed true if the curvature or field $|F|$ has growth at most $\left(r^{2} \log r\right)^{-1}$ [4]. We prove that finite energy $\int_{\mathbf{R}^{4}}|F|^{2} d x$ is sufficient. We prove general $n$-dimensional results. We assume throughout the paper that $G$ is a compact Lie group with bi-invariant metric and $\mathrm{g}$ is the Lie algebra for $G$.

Theorem. Let $A_{j} \in L_{1, \text { loc }}^{n / 2}\left(\mathbf{R}^{n}, \mathfrak{g}\right), j=1,2, \ldots, n>2$ and let $F=F_{A}=d A+A \wedge A$ be the curvature of the connection $d+A$. If $n$ is even, $n \neq 2$, and $\int_{\mathbf{R}^{n}}|F|^{n / 2} d x<\infty$, then the chern number arising from a representation $\rho: G \rightarrow \mathrm{SU}(N)$ is integral.

The proof is somewhat lengthy, and could be shortened considerably for the case $A_{j}$ smooth. However, it seemed worthwhile to treat the most general case, $A_{j} \in L_{1, \text { loc }}^{n / 2}$, for the purpose of completeness. The various technical theorems we use to handle non-smooth $A_{j}$ have interesting features and possible applications elsewhere. The main idea of the proof is to choose a good gauge near $(\infty)$. This relies on an earlier theorem on the existence of good (Coulomb) gauges [5]. The idea for the proof arose from conversations with $\mathrm{L}$. M. Sibner about the removable singularities theorem in dimension $3[6]$. 


\section{Sobolev connections and Chern Number}

Let $P$ be a principal bundle over a compact Riemannian $n$-manifold $M$ with structure group $G$, Lie algebra $\mathrm{g}$. Recall that the class of smooth connections is an affine space, described by choosing any base connection $D_{0}$ and setting

$$
\mathscr{A}(P)=\left\{D=D_{0}+A: A \in C^{\infty}\left(T^{*} M \otimes \operatorname{Ad} P\right)\right\} .
$$

We shall use the bundles $T^{*} M \otimes \operatorname{Ad} P=\eta_{1}(P)$ and $T^{*} M \wedge T^{*} M \otimes \operatorname{Ad} P=\eta_{2}(P)$ of Lie algebra-valued one and two forms, so often we use the notation $\eta_{1}(P)$ and $\eta_{2}(P)$. The space of Sobolev connections is

$$
\mathscr{A}_{k}^{p}(P)=\left\{D_{0}+A: A \in L_{k}^{p}\left(\eta_{1}(P)\right)\right\} .
$$

Here $L_{k}^{p}(\eta)$ denotes the Banach space of sections of $\eta$ with partials up through order $k$ in $L^{p}$. There is an equivalent local description. If $\mathscr{U}_{\alpha}$ is a cover of $M$ and $\rho_{\alpha}: P \mid \mathscr{U}_{\alpha} \sim$ $G \times \mathscr{U}_{\alpha}$ are smooth local trivializations, then

$$
D \mid \mathscr{U}_{\alpha} \sim d+A_{\alpha},
$$

where $A_{\alpha} \in L_{k}^{p}\left(\mathscr{U}_{\alpha}, \mathbf{R}^{n} \otimes \mathfrak{g}\right)$. It is a well-known fact that $\mathscr{A}(P)$ is dense in $\mathscr{A}_{k}^{p}(P)$.

The curvature of a smooth connection $D$ is a smooth section $F(D)$ of the bundle $\eta_{2}(P)$ of Lie algebra-valued two forms. In local coordinates $F \mid \mathscr{U}_{\alpha}=d A_{\alpha}+A_{\alpha} \wedge A_{\alpha}$. From the local description, we easily see the following lemma:

Lemma 1.1. The curvature map $F: \mathscr{A} \rightarrow C^{\infty}\left(\eta_{2}(P)\right)$ extends to a smooth (in fact quadratic) map $F: \mathscr{A}_{1}^{p} \cap \mathscr{A}_{0}^{2 p} \rightarrow L_{0}^{p}\left(\eta_{2}(P)\right)$. If $2 p \geqq n=\operatorname{dim} M$, then $\mathscr{A}_{1}^{p} \subset \mathscr{A}_{0}^{2 p}$ and $F: \mathscr{A}_{1}^{p} \rightarrow L_{0}^{p}\left(\eta_{2}(P)\right)$.

Now let $\Lambda^{n}(M)=\Lambda^{n}$ be the bundle of $n$-forms over $M$. A smooth bundle map $\Psi: \eta \rightarrow \Lambda^{n}$ is said to be a homogeneous polynomial of degree $q$ if $\Psi_{x}\left(\eta_{x}\right)=$ $h_{x}\left(\eta_{x} \otimes \eta_{x} \otimes \cdots \otimes \eta_{x}\right)$, where $h:(\otimes \eta) \rightarrow \Lambda^{n}$ is linear.

This thinking leads us to the elementary observation:

Proposition 1.2. Let $\Psi: \eta_{2}(P) \rightarrow \Lambda_{n}$ be a homogeneous polynomial map of degree $p$. Then the induced map $\bar{\Psi}: \mathscr{A} \rightarrow \mathbf{R}$ given by

$$
\bar{\Psi}(D)=\int_{M} \Psi(F(D))
$$

extends to a smooth map $\Psi: \mathscr{A}_{1}^{p}(P) \cap \mathscr{A}^{2 p}(P) \rightarrow \mathbf{R}$.

Proof. Note $\Psi$ factors into a composition

$$
\mathscr{A}_{1}^{p}(P) \cap \mathscr{A}^{2 p}(P) \stackrel{F}{\rightarrow} L_{0}^{p}\left(\eta_{2}\right) \stackrel{\Psi}{\rightarrow} L_{0}^{1}\left(\Lambda^{n}\right) \stackrel{\int}{\rightarrow} \mathbf{R} .
$$

Each piece is smooth. The map $\bar{\Psi}$ itself is polynomial. If $\Psi$ is merely homogeneous of order $p,\left(\Psi_{x}\left(c \eta_{x}\right)=c^{p} \Psi_{x}\left(\eta_{x}\right), c>0\right)$, then $\bar{\Psi}$ is $C^{k}$ for $k$ any integer less than $p$.

Corollary 1.3. If $\bar{\Psi}$ takes on a constant value $\gamma$ on $\mathscr{A}(P)$, then it has the value $\gamma$ on every connection in $\mathscr{A}_{1}^{p}(P) \cap \mathscr{A}_{0}^{2 p}(P)$.

Proof. This follows from the density of $\mathscr{A}(P)$ in $\mathscr{A}_{1}^{p}(P) \cap \mathscr{A}_{0}^{2 p}(P)$ and the continuity 
of $\Psi$. For $m<n / 2$, the following can be compared to Sedlacek's result [7].

Corollary 1.4. If $\rho: G \rightarrow \mathrm{SU}(N)$ is a representation, and $\omega$ a smooth $n-2 m$ form on $M$, then the characteristic class cupped with $\omega$ and evaluated on $[M], c_{m}(\omega)$, which is a multiple of

$$
\int_{M}\left(\operatorname{tr} \rho(F)^{m}\right) \wedge \omega
$$

is constant on $\mathscr{A}_{1}^{m} \cap \mathscr{A}^{2 m}$. In particular, if $n$ is even and $m=n / 2$,

$$
\int_{M} \operatorname{tr} \rho(F)^{n / 2}
$$

is a fixed integer times the appropriate dimensional constant on $\mathscr{A}_{1}^{n / 2}$.

Proof. Here $\Psi$ is given by $\left(\operatorname{tr} \rho(F)^{m}\right) \wedge \omega$, which is polynomial. Since $L_{1}^{n / 2} \subset L^{n}$, we may replace $\mathscr{A}_{1}^{n / 2} \cap \mathscr{A}^{n}$ by $\mathscr{A}_{1}^{n / 2}$.

Corollary 1.5. Let $\mathbf{S}: S^{2 m}-\{\infty\} \rightarrow \mathbf{R}^{n} \cdot \mathbf{S}^{-1}: \mathbf{R}^{2 m} \rightarrow S^{2 m}-\{0\}$ be the usual stereographic projection and its inverse. Let $D \in \mathscr{A}_{1}^{m}$ be a connection in a principal bundle $P$ on $S^{2 m}$, and let the connection $d+A$ on $\mathbf{R}^{2 m}$

$$
d+A=\left(\mathbf{S}^{-1}\right)^{*} D
$$

be obtained by pull-back of D. Then the chern number

$$
c_{m}=\left(\frac{1}{2 \pi i}\right)^{m} \frac{(-1)^{m+1}}{m} \int_{\mathbf{R}^{2 m}} \operatorname{tr}\left(\rho(F)^{m}\right)
$$

is integral, where $F=F(d+A)=d A+A \wedge A$.

Proof. Due to the way chern number is defined, on $S^{n}$

$$
c_{m}=\left(\frac{1}{2 \pi i}\right)^{m} \frac{(-1)^{m+1}}{m} \int_{s^{2 m}} \operatorname{tr}\left(\rho(F(D))^{m}\right),
$$

which is integral by the previous corollary.

\section{Construction of the Bundles on $\mathbf{S}^{n}$}

Recall that if $M^{\prime}$ is a non-compact manifold, by $L_{k, \text { loc }}^{p}\left(M^{\prime}\right)$ we mean the Frechet space of functions whose restriction to any compact domain $\overline{\mathscr{U}} \subset M^{\prime}$ lie in $L_{k}^{p}(\overline{\mathscr{U}})$. Let $B^{\prime}=\left\{x \in \mathbf{R}^{n}, 0<|x| \leqq 1\right\}$. In Sect. 4, we obtain the following result as Corollary 4.6.

Theorem 2.1. There exists $\varepsilon=\varepsilon(G, n)>0$ such that if $D \in \mathscr{A}_{1, \text { loc }}^{n / 2}\left(B^{\prime}\right)$ and

$$
\int_{0<|x| \leqq 1}|F(D)|^{n / 2} d x=\lim _{r \rightarrow 0} \int_{r \leqq|x| \leqq 1}|F(D)|^{n / 2} d x<\varepsilon,
$$

then there exists $s \in L_{2, \mathrm{loc}}^{n / 2}\left(B^{n}, G\right)$ such that in the gauge

$$
s^{-1} D s=d+s^{-1} d s+s^{-1} A s=d+\tilde{A},
$$

we have $A \in L_{1}^{n / 2}\left(B, \mathbf{R}^{n} \otimes \mathfrak{g}\right)$. Also

$$
\|A\|_{L_{1}^{n / 2}(B)} \leqq c(n, G)\|F(D)\|_{L^{n / 2}(B)}
$$


Moreover, s may be chosen smooth on $\{x: 0<\rho \leqq|x| \leqq 1-\rho<1\}$.

We now describe how to pass from this technical result to our main result. We state the result for $\mathbf{R}^{n}$, although it, of course, applies to any conformal equivalent of a compact manifold with a finite number of points omitted.

Corollary 2.2. Let $A \in L_{1, \text { loc }}^{n / 2}\left(\mathbf{R}^{n}, \mathbf{R}^{n} \otimes \mathfrak{g}\right)$, and $\int_{\mathbf{R}^{n}}\left|F_{A}\right|^{n / 2} d x<\infty$. Then the connection $D=d+A$ is gauge equivalent to the pull-back via the inverse stereographic projection of a connection $D \in \mathscr{A}_{1}^{n / 2}(P)$, for some smooth principle bundle on $S^{n}$.

Proof. Since $\int_{\mathbf{R}^{n}}\left|F_{A}\right|^{n / 2} d x<\infty$, we can choose a $K<\infty$ such that $\int_{|x| \geqq K}\left|F_{A}\right|^{n / 2} d x<\varepsilon$. Cover $S^{n}$ with ${ }^{\boldsymbol{R}^{n}}$ two coordinate charts, $\mathscr{U}_{1}=\mathbf{S}^{-1}(x:|x| \leqq 4 K)$ and $\mathscr{U}_{2}=\mathbf{S}^{-1}(x:|x| \geqq$ $K) \cup\{\infty\}$. Parametrize $B=\mathscr{U}_{2}, B^{\prime}=\mathbf{S}^{-1}(x:|x| \geqq K)$ conformally in the obvious way. Now apply Theorem 2.1 to the connection $d+A$, which is now regarded as a connection $d+A^{\prime}$ on $B^{\prime}$. By conformal invariance.

$$
\int_{|x| \geqq K}\left|F_{A}\right|^{2} d x=\int_{|x| \leqq 1}\left|F_{A^{\prime}}\right|^{2} d x<\varepsilon .
$$

The overlap between two charts is in the coordinates in $\mathscr{U}_{2}=B$ (letting $\tilde{\mathscr{U}}_{2}=$ $\left\{|x| \leqq \frac{1}{2}\right\}$.

$$
\tilde{\mathscr{U}}_{2} \cap \mathscr{U}_{1}=\left\{x \in B^{\prime}: \frac{1}{4} \leqq|x| \leqq \frac{1}{2}\right\} .
$$

The map $s: B^{\prime} \rightarrow G$ restricts to the overlap function $g_{12}: \widetilde{\mathscr{U}}_{2} \cap \mathscr{U}_{1} \rightarrow G$, and can be chosen smooth in this range $\left\{x: \frac{1}{4} \leqq|x| \leqq \frac{1}{2}\right\}\left(\rho=\frac{1}{4}\right)$. This map $g_{12}=s$ describes the principal bundle $P$ on $S^{n}$. Since we started with $A \mid \mathscr{U}_{1}$ in $L_{1}^{n / 2}$, and by coordinate change we obtain in $\tilde{\mathscr{U}}_{2}$

$$
\tilde{A}=s^{-1} d s+s^{-1}\left(A^{\prime} \mid \tilde{\mathscr{U}}_{2}\right) s
$$

in $L_{1}^{n / 2}$, we have a local description of a connection in $\mathscr{A}_{1}^{n / 2}(P)$.

Corollary 2.3. If $A \in L_{1, \text { loc }}^{n / 2}\left(\mathbf{R}^{n}, \mathbf{R}^{n} \otimes \mathfrak{g}\right),(n=2 m)$ and $\lim _{r \rightarrow \infty} \int_{|x| \leqq r}\left|F_{A}\right|^{n / 2} d x<\infty$, then

$$
c_{m}=\left(\frac{1}{2 \pi i}\right)^{m} \frac{(-1)^{m+1}}{m} \int_{\mathbf{R}^{n}} \operatorname{tr}(\rho(F))^{m} d x
$$

is an integer.

Proof. By the preceding corollary, the connection $d+A$ is obtained via a pull-back from an $\mathscr{A}_{1}^{n / 2}(P)$ connection on $S^{n}$. By Corollary $1.5, c_{n / 2}$ is integral.

\section{A Density Theorem}

In Sect. 4, we obtain a map $u: B^{\prime} \rightarrow G, u \in L_{2, \text { loc }}^{n / 2}\left(B^{\prime}, G\right)$. Now $u$ is not necessarily continuous (it is if $u \in L_{2, \text { loc }}^{p}\left(B^{\prime}, G\right)$ for any $p>n / 2$ ). We wish to approximate $u$ by a map which is smooth in an annullus $0<\rho \leqq|x| \leqq 1-\rho<1$. To do this we mimic the proof of the approximation of $L_{1}^{2}$ maps from surfaces by Schoen and Uhlenbeck [8]. The slight technical difference is that we wish to keep the approximation fixed near the boundary. 
For the following, $G$ is any compact manifold isometrically immersed in $\mathbf{R}^{k}$ (i.e., the Lie group structure is irrelevant). In our case, we consider $\rho: G \subset \mathrm{SU}(N) \subset$ $\mathbf{C}^{N} \times \mathbf{C}^{N}=\mathbf{R}^{k}\left(k=2 N^{2}\right)$. Let $\mathcal{O}_{\delta}$ be the set of points at distance $\delta$ from $G$. If $\delta$ is sufficiently small, the nearest point projection from $\mathbf{R}^{k}$ to $G$ is well-defined and smooth on $\mathcal{O}_{\delta}$. Call this $\Pi: \mathcal{O}_{\delta} \rightarrow G$. Given any domain $\Omega \subset \mathbf{R}^{n}$, define

$$
L_{k}^{p}(\Omega, G)=\left\{u \in L_{k}^{p}\left(\Omega, \mathbf{R}^{k}\right): u(x) \in G \quad \text { a.e. }\right\} .
$$

Let $\Omega_{h}=\left\{x \in \Omega\right.$ :dist $\left.\left(x, \mathbf{R}^{n}-\Omega\right) \leqq h\right\}$. Also, let $\varphi$ be any positive, smooth bump function with compact support in the unit ball, $\int_{\mathbf{R}^{n}} \varphi(y) d y=1$. Given $u \in L_{k}^{p}\left(\Omega, \mathbf{R}^{k}\right)$, $x \in \Omega_{n}$

$$
u^{h}(x)=\int_{|x-y| \leqq h} u(x+h y) \varphi(y) d y .
$$

It is well-known that $u^{h}$ is smooth on $\Omega_{h}$. However, $u^{h}(x) \notin G$.

Lemma 3.1. There exists $\varepsilon_{0}=\varepsilon_{0}(n, G)$ such that if $\int_{B_{h}(x)}|d u|^{n}(y) d y \leqq \varepsilon \leqq \varepsilon_{0}$, then the mollified function $u^{h}$ has the property $\operatorname{dist}\left(u^{h}(x), G\right)<K \varepsilon^{1 / n}$.

Proof. The condition given in Schoen and Uhlenbeck [9.3.2] is $h^{-n+2} \int_{B_{h}(x)}|d u|^{2} d y \leqq$ $c \varepsilon^{1 / 2}$, which is implied by our assumption and the Hölder inequality.

Theorem 3.2. Let $u \in L_{1}^{n}(\Omega, G)$, and $\Omega$ be a compact domain in $\mathbf{R}^{n}$ with smooth boundary. Then given $\mu>0, d>0$ there exists $\tilde{u} \in L_{1}^{n}(\Omega, G), \tilde{u}=u$ on $\overline{\Omega-\Omega_{d}}$, $\tilde{u} \mid \Omega_{2 d} \in C^{\infty}\left(\Omega_{2 d}, G\right)$ and $\|\tilde{u}-u\|_{L^{n}\left(\Omega, \mathbf{R}^{k}\right)} \leqq \mu$. If $\mu \in L_{2}^{n / 2}(\Omega, G)$, we may find $\tilde{u}$ with $\|\tilde{u}-u\|_{L_{2}^{n / 2}\left(\Omega, \mathbf{R}^{k}\right)} \leqq \mu$.

Proof. For $x \in \Omega_{d}$, we have that $\varepsilon(x, h)=\int_{|x-y| \leqq h}|d u|^{n} d x$ is a continuous family (in $\left.h\right)$ of continuous functions on $\Omega_{d}$ decreasing to 0 . Therefore $\varepsilon(n)=\max \varepsilon(x, h) \rightarrow 0$. So for $h$ sufficiently small, $\operatorname{dist}\left(u^{h}(x), G\right) \leqq K(\varepsilon(h))^{1 / n} \rightarrow 0$. We observe that the proof that $\lim _{h \rightarrow 0} u_{h}^{*}=u$ in $L_{1}^{n}(\Omega)$ is exactly as in the classical case $f \equiv 1$, once we show that the linear map $v \rightarrow v_{h}^{*}$ on $L_{1}^{n}$ satisfies $\left\|v_{h}^{*}\right\|_{L_{1}^{n}(\Omega)} \leqq \bar{K}\|v\|_{L_{1}^{n}(\Omega)}$. But

$$
\begin{aligned}
\left\|v_{h}^{*}\right\|_{L^{n}(\Omega)}^{n} & \leqq \int \varphi(y) \int|v(x-h f(x) y)|^{n} d x d y \\
& \leqq \int \varphi(y) K \int|v(z)|^{n} d z d y \\
& =K\|v\|_{L^{n(\Omega)}}^{n} .
\end{aligned}
$$

Here $K=\max _{y, x, h \leqq d}\left(\operatorname{det} \frac{\partial(x-h f(x) y)}{\partial x}\right)^{-1}$. By differentiating (3.2), we obtain

$$
\left|d\left(v_{h}^{*}\right)(x)\right| \leqq\left.|| d v\right|_{h} ^{*}(x)|+h \max | d f \mid\left(\left|v_{h}^{*}(x)\right|\right) .
$$

From this we get (using (3.3) again on $d v$ and $|v|$ )

$$
\left\|d\left(v_{h}^{*}\right)\right\|_{L^{n}(\Omega)} \leqq K^{1 / n}\|d v\|_{L^{n}(\Omega)}+K^{1 / n} h \max |d f|\|v\|_{L^{n}(\Omega)} .
$$



already

Finally, we show $\left\|u_{h}^{*}-\tilde{u}_{h}\right\|_{L_{1}^{n}(\Omega)} \rightarrow 0$, (which completes the proof). We have

$$
\max _{x \in \Omega}\left|\tilde{u}_{h}(x)-u_{h}^{*}(x)\right| \leqq K_{2}(\varepsilon(n))^{1 / n} \rightarrow 0
$$

from Lemma 3.1. By the chain rule

$$
\begin{aligned}
\left|d \tilde{u}_{h}(x)-d u_{h}^{*}(x)\right| & \leqq\left|d \Pi\left(u_{h}^{*}(x)\right)-I\right|\left|d u_{h}^{*}(x)\right| \\
& \leqq K_{3}(\varepsilon(h))^{1 / n}\left|d u_{h}^{*}(x)\right| .
\end{aligned}
$$

Integrating both inequalities completes the proof. The proof for $L_{2}^{n / 2}(\Omega)$ simply involves one more differentiation.

Note that this would be the first step in a proof that an $L_{2}^{n / 2}$ bundle is "equivalent" to a smooth bundle. See Sedlacek [7] for a situation where such a theorem might be useful.

\section{Coulomb Gauges}

The theorem we wish to extend is the following theorem, proved in [5].

Theorem 4.1. There exists $\varepsilon_{0}=\varepsilon(n, G)>0$ and $K_{0}=K_{0}(n, G)$ such that if $A \in L_{1}^{n / 2}\left(B^{n}, \mathbf{R}^{n} \times \mathfrak{g}\right)$ and $\int_{B^{n}}\left|F_{A}\right|^{n / 2} d x<\varepsilon$, then there exists $s \in L_{2}^{n / 2}\left(B^{n}, G\right)$ such that $\tilde{A}=s^{-1} d s+s^{-1}$ As satisfies
a) $d^{*} A=0$
b) $\int_{B^{n}}\left(|d \tilde{A}|^{n / 2}+|\tilde{A}|^{n / 2}\right) d x \leqq K_{1} \int_{B^{n}}\left|F_{A}\right|^{n / 2} d x$.

We use this above theorem and the following compactness theorem where $m=n-1$ [5].

Theorem 4.2. If $2 p>m, M=M^{m}$ is a compact manifold, and $D_{i}$ is a sequence of connections with $\int_{M}\left|F\left(D_{i}\right)\right|^{p} d x<b$, then a subsequence of the $D_{i}$ is gauge equivalent to $a$ sequence $D_{i}$ which converges weakly in the space of $L_{1}^{p}$ connections.

The general techniques give an immediate corollary, which is what we shall use.

Corollary 4.3. If $2 p>m$ and $M=M^{m}$ a compact manifold, then there exists an $\varepsilon_{1}(p, M, G)>0$ such that if $D$ is a connection with $\int_{M}|F(D)|^{p} d x<\varepsilon_{1}$, then there exists a flat connection d on $M$ such that $D$ is gauge equivalent to $D^{\prime}$ with

$$
\left\|d-D^{\prime}\right\|_{L_{1}^{p}(M)}^{p} \leqq K_{1} \int_{M}|F(D)|^{p} d \mu .
$$

Proof. By the weak compactness theorem we may assume that for $\varepsilon_{1}$ small, $D^{\prime}$ is weakly close to a flat connection and close in $L_{0}^{q}$ for $m<q<2 p$. We may then find a Coulomb gauge for $D^{\prime}$ by solving

$$
d^{*}\left(g^{-1} d g+g^{-1}\left(d-D^{\prime}\right) g\right)=0
$$


in the space $g \in L_{1}^{q}(M, G) \subset C^{0}(M, G)$ by means of the implicit function theorem. In the new gauge $D^{\prime}=d+A$, where $A$ is small in $L_{0}^{q}, q>m, d^{*} A=0$, and it is easily seen that the $L_{1}^{p}$ norm of $A$ is bounded by a multiple of $\int_{M}|F(D)|^{p} d \mu$ by the usual techniques of standard elliptic theory.

We now prove our main extension lemma.

Lemma 4.4. There exists $\varepsilon_{2}=\varepsilon_{2}(n, G)$ such that if $A \in L_{1}^{n / 2}\left(\left\{x: \rho_{1} \leqq x \leqq \rho_{2}\right\}, \mathbf{R}^{n} \times \mathfrak{g}\right)$ and $\rho_{1}^{-1} \int_{|x|=\rho_{1}}\left|F_{A}\right|^{n / 2} d \mu=\varepsilon<\varepsilon_{2}$, then $A$ is gauge equivalent to a connection $\tilde{A}$ which extends to a connection (again called $\tilde{A}$ ), $\tilde{A} \in L_{1}^{n / 2}\left(|x| \leqq \rho_{2}, \mathbf{R}^{n} \times \mathfrak{g}\right.$ ) and $\int_{|x| \leqq \rho_{1}}\left|F_{A}\right|^{n / 2}$ $d x \leqq K_{2} \varepsilon$.

Proof. By conformal invariance, we may assume $\rho_{1}=1, \rho_{2}=\rho>1$. Let $i(x)=x$ imbed $S^{n-1} \subset \mathbf{R}^{n}$ and let $i^{*} A=A^{*}$ be the pull-back Sobolev connection in a bundle over $S^{n-1} \cdot \int_{S^{n-1}}\left|F_{A^{*}}\right|^{n / 2} * 1=\int_{S^{n-1}}\left|i^{*} F_{A}\right|^{n / 2} * 1 \leqq \varepsilon_{2}$. By Corollary 4.3 , with $p=n / 2$, $m=n-1, \stackrel{S^{n-1}}{M}=S^{n-1}$, if $\varepsilon_{2}$ is sufficiently small $d+A^{*}$ is gauge equivalent to $d+A^{* *}$, where

$$
\left\|A^{* *}\right\|_{L_{1}^{n / 2}\left(S^{n-1}\right)}^{n / 2} \leqq K_{1} \int_{S^{n-1}}\left|F_{A}\right|^{n / 2} * 1 \leqq K_{1} \varepsilon_{2} .
$$

Since $C^{\infty}$ is dense in $L_{1}^{n / 2}\left(S^{n-1}\right)$ we may assume the gauge transformation $s$ is smooth. Extend it to all of the annulus by $s(x)=s\left(\frac{x}{|x|}\right)$ and transform $A$ via it. In the new gauge $i^{*} A$, the tangential part of $A$ is small on $S^{n-1}$.

Let the normal part of $A, A_{r} \in L_{1}^{n / 2}\left(S^{n-1}, \mathfrak{g}\right)$, be approximated (to within $(\varepsilon)^{2 / n}$ ) by a smooth $C^{\infty}$ section $g_{r}$. Define a second smooth gauge transformation on $1 \leqq|x| \leqq \rho$ by $g(1, \theta)=I, \partial g / \partial r(1, \theta)=-g_{r}$ and $g(r, \theta)$ is geodesic in $G$ as a function of $r$. Gauge transform again. Now we have the norm in this gauge

$$
\left\|A \mid S^{n-1}\right\|_{L_{1}^{n / 2}\left(S^{n-1} \cdot \mathbf{R}^{n} \times g\right)}^{n / 2} \leqq K_{2}^{\prime} \varepsilon .
$$

Extend (in this gauge) by setting

$$
A(x)=f(x) A\left(\frac{x}{|x|}\right)
$$

for $f(x)$ a smooth function, $f(x)=0$ for $|x| \leqq 1 / 2$ and $f(x)=1$ for $|x| \geqq 3 / 4$. Now

$$
\begin{aligned}
\int_{|x| \leqq 1}\left|F_{A}\right|^{n / 2} d x & \leqq C_{1}\left\|A \mid B^{n}\right\|_{L_{1}^{n / 2}\left(B^{n}\right)}^{n / 2} \\
& \leqq C_{1} C_{2}\left\|A \mid S^{n-1}\right\|_{L_{1}^{n / 2}\left(S^{n-1}\right)}^{n / 2} \leqq C_{1} C_{2} K_{2}^{\prime} \varepsilon
\end{aligned}
$$

This completes the proof, with $K_{2}=C_{1} C_{2} K_{2}^{\prime}$. The main technical difficulty is next solved. 
Theorem 4.5. There exists an $\varepsilon_{3}>0$ and $K_{3}<\infty$ such that if $A \in L_{1, \text { loc }}^{n / 2}\left(B^{\prime}, \mathbf{R}^{n} \times \mathfrak{g}\right)$ and $\int_{B^{n}}\left|F_{A}\right|^{n / 2} d x<\varepsilon_{3}$, then there exists a gauge transformation $g \in L_{2, \text { loc }}^{n / 2}\left(B^{\prime}, G\right)$ such that the gauge transformed connection

$$
\tilde{A}=g^{-1} d g+g^{-1} A g \in L_{1}^{n / 2}\left(B^{\prime}, \mathbf{R}^{n} \times \mathfrak{g}\right) .
$$

Moreover $d^{*} \tilde{A}=0$ and $\|\tilde{A}\|_{L_{1}^{n / 2}\left(B^{n}\right)}^{n / 2} \leqq K_{3} \int_{B^{n}}\left|F_{\tilde{A}}\right|^{n / 2} d x$.

Proof. Choose a sequence of radii $\rho_{i} \rightarrow 0$ such that $A \mid\left\{|x|=\rho_{i}\right\} \in L_{1}^{n / 2}$ and $\rho_{i} \int_{|x|=\rho_{i}}\left|F_{A}\right|^{n / 2} d x=\varepsilon_{i} \rightarrow 0$. Let $A_{i}$ be the extension of $A_{i}$ gauge equivalent to $A \mid\left\{x: \rho_{i} \leqq|x| \leqq 1\right\}$ given by Lemma 4.4. We see immediately that

$$
\int_{|x| \leqq 1}\left|F_{A_{i}}\right|^{n / 2} d x \leqq \int_{|x| \leqq \rho_{i}}\left|F_{A_{i}}\right|^{n / 2} d x+\int_{|x| \leqq 1}\left|F_{A}\right|^{n / 2} d x \leqq K_{2} \varepsilon_{i}+\varepsilon_{3} .
$$

Apply Theorem 4.1, which gives a gauge equivalent $\tilde{A}_{i}$ which can be estimated by $\left\|\widetilde{A}_{i}\right\|_{L_{1}^{n / 2}\left(B_{n}\right)}^{n / 2} \leqq K_{0}\left(K_{2} \varepsilon_{i}+\varepsilon_{3}\right)$. Here $d^{*} \widetilde{A}_{i}=0$.

First we may choose a weakly convergent subsequence $\widetilde{A}_{i}-\tilde{A} \in L_{1}^{n / 2}\left(B^{n}, \mathbf{R}^{n} \times \mathfrak{g}\right)$. The weak convergence implies $d^{*} \tilde{A}=0$, and the inequality

$$
\|A\|_{L_{1}^{n / 2}\left(B^{n}\right)}^{n / 2} \leqq K_{0} \varepsilon_{3} .
$$

Let $\mathscr{C}_{i}=\left\{x: \rho_{i} \leqq|x| \leqq 1\right\}$ and $\tilde{g}_{i} \in L_{1}^{n / 2}\left(\mathscr{C}_{i}, G\right)$ be the composition of the several gauge transformations constructed by Lemma 4.4 and Theorem 4.5 relating $A \mid \mathscr{C}_{i}$ and $\widetilde{A}_{i}$. Then

$$
\tilde{A}_{i}=\tilde{g}_{i}^{-1} \circ d \tilde{g}_{i}+\tilde{g}_{i}^{-1} A \tilde{g}_{i} .
$$

If we fix $j$, this equation holds on $\mathscr{C}_{j}$ for $i \geqq j$. Since $A \mid \mathscr{C}_{j} \in L_{1}^{n / 2}\left(\mathscr{C}_{j}, \mathbf{R}^{n} \times \mathfrak{g}\right)$ and on $\mathscr{C}_{j}$, $i \geqq j$, we have for almost all $x$

$$
\begin{aligned}
\left|d \tilde{g}_{i}\right| & =\left|\tilde{g}_{i} \tilde{A}_{i}-A \tilde{g}_{i}\right| \leqq\left|\tilde{A}_{i}\right|+|A|, \\
\left|d^{2} \tilde{g}_{i}\right| & =\left|\tilde{g}_{i} d \tilde{A}_{i}-d A \tilde{g}_{i}+d \tilde{g}_{i} \tilde{A}_{i}-\tilde{A} d g_{i}\right| \\
& \leqq\left|d \tilde{A}_{i}\right|+|d A|+\left|d \tilde{g}_{i}\right|\left|\tilde{A}_{i}\right|+|A|\left|d \tilde{g}_{i}\right| .
\end{aligned}
$$

From the first inequality and the Sobolev theorem we find for $i \geqq j$

$$
\begin{aligned}
\left\|\tilde{g}_{i}\right\|_{L_{1}^{n}\left(\mathscr{C}_{j}\right)} & \leqq\left\|\tilde{A}_{i}\right\|_{L^{n}\left(B^{n}\right)}+\left\|A \mid \mathscr{C}_{j}\right\|_{L^{n}\left(\mathscr{C}_{j}\right)}+k \\
& \leqq c^{j}\left(\left\|\tilde{A}_{i}\right\|_{L_{1}^{n / 2}\left(B^{n}\right)}+\left\|A \mid \mathscr{C}_{j}\right\|_{L_{1}^{n / 2}\left(\mathscr{C}_{j}\right)}\right)+k
\end{aligned}
$$

From this estimate, the second almost everywhere inequality and the Holder inequality we obtain

$$
\left\|\tilde{g}_{i}\right\|_{L_{2}^{n / 2}\left(\mathscr{C}_{j}\right)} \leqq\left\|\tilde{A}_{i}\right\|_{L_{1}^{n / 2}\left(B^{n}\right)}+\left\|A \mid \mathscr{C}_{j}\right\|_{L_{1}^{n / 2}\left(B^{n}\right)}+\left\|\tilde{g}_{i}\right\|_{L_{1}^{n}\left(\mathscr{C}_{j}\right)}\left(\left\|\tilde{A}_{i}\right\|_{L^{n}\left(B^{n}\right)}+\left\|A \mid \mathscr{C}_{j}\right\|_{L^{n}\left(\mathscr{C}_{j}\right)}\right) .
$$

By a diagonal argument, we extract a subsequence which converges weakly to a limit $g$ in $L_{2}^{n / 2}\left(\mathscr{C}_{j}, G\right)$ for all $j$. This produces $g \in L_{2, \text { loc }}^{n / 2}\left(B^{n}, G\right)$. Since the weak limit implies almost everywhere convergence for $\tilde{A}_{i}$ and $\tilde{g}_{i}$ we obtain

$$
\tilde{A}=g^{-1} d g+g^{-1} A g \text {. }
$$


This completes the proof.

We obtain Theorem 2.1 as a simple corollary of this theorem and Theorem 3.2.

Corollary 4.6. In the statement of Theorem 4.5, if we relax the condition that $d^{*} \tilde{A}=0$, we may assume $g \mid\{x: \rho \leqq|x| \leqq 1-\rho\} \in C^{\infty}$.

Proof. Let $\Omega=\{x: \rho / 2 \leqq|x| \leqq 1\}$ and $d=\rho / 4$. We may approximate $g \in L_{2}^{n / 2}(\Omega, G)$ as closely as we like by a function which is smooth on $\Omega_{d}$. If the approximation is close enough, the inequalities remain valid, although $d^{*} \tilde{A}$ will no longer be zero in the new gauge.

\section{References}

1. Uhlenbeck, K.: Removable singularities in Yang-Mills fields. Commun. Math. Phys. 83, 11-29(1982)

2. Parker, T.: Gauge theories on four dimensional Riemannian manifolds. Commun. Math. Phys. 85, 563-602 (1982)

3. Witten, E.: Instantons the quark model, and the $1 / \mathrm{N}$ expansion. Nucl. Phys. B 149, 285-320 (1979)

4. Schlafly, R.: A Chern number for gauge fields on $\mathbb{R}^{4}$. J. Math. Phys. 23, 1379-1394 (1982)

5. Uhlenbeck, K.: Connections with $L^{p}$ bounds on curvature. Commun. Math. Phys. 83, 31-42 (1982)

6. Sibner, L. M., Sibner, R. J.: Removeable singularities of coupled Yang-Mills fields in $\mathbb{R}^{3}$, Commun. Math. Phys. 93, 1-17 (1984)

7. Sedlacek, S.: A direct method for minimizing the Yang-Mills functional over 4 manifolds. Commun. Math. Phys. 86, 515-528 (1982)

8. Schoen, R., Uhlenbeck, K.: J. Differ. Geom. 18, 253-268 (1983)

9. Schoen, R., Uhlenbeck, K.: J. Differ. Geom. 17, 307-335 (1982)

Communicated by S.-T. Yau

Received October 1, 1982, in revised form March 22, 1985 
